\title{
Computer-aided learning programmes in teaching dental students
}

\author{
Are computer-aided learning programmes effective in teaching dental \\ students?
}

\begin{abstract}
Rosenberg H, Grad HA, Matear DW. The effectiveness of computer-aided, self-instructional programs in dental education: a systematic review of the literature. J Dent Educ 2003; 67:524532
\end{abstract}

Data sources Sources were MEDLINE, the Cochrane Library, Embase, ERIC (Educational Resources Information Centre), CINHAL (Cumulative Index to Nursing and Allied Health), LISA (Library and Information Science Abstracts), Psycinfo (Psychological Information) and IPA (International Pharmaceutical Abstracts).

Study selection Randomised controlled trials (RCT) that compared computer-aided learning (CAL) programmes with any other method of instruction were considered. Only studies within dentistry were included. The quality of studies was assessed using a checklist.

Data extraction and synthesis Both qualitative and quantitative outcomes from studies were recorded, and qualitative synthesis of the results was undertaken because of the diverse nature of the outcomes. Results Twelve studies were identified (five in endodontics, three in orthodontics and one each in oral anatomy, restorative, geriatric and prosthetic dentistry).

Conclusions For the most part, CAL is either more effective than or equally effective as other methods of education.

\section{Commentary}

This paper examines the effectiveness of CAL programmes in the dental literature. The authors found, from their search of the literature, that 27 studies met their strict criteria for consideration in the review. The main and most important criterion was whether the report was of a RCT. These selected studies showed that CAL is as effective as other methods of teaching: the authors consider it a worthwhile addition to the undergraduate curriculum.

The use of CAL provokes much discussion within dental institutions as to whether it is effective in the teaching of undergraduates. Furthermore, as clinical scientists, we feel comfortable with research studies that look at two groups of individuals who receive learning materials presented in two different forms. The experimental group receives the CAL programme whereas the control group receives the same information presented in a traditional form, such as a lecture, small-group teaching or self instruction without the use of computers. This review has applied the same rigour to the evaluation of CAL that would be applied to a clinical RCT. Whether the RCT is the correct vehicle for assessing the effectiveness of CAL remains open to debate, nevertheless.

Learning is a complex process and the assessment of how students have assimilated knowledge from the learning materials presented to them will also be complex. All the studies selected in this paper followed the approach of a RCT and the outcome was to determine whether a student successfully passed a subsequent examination. In the discussion, the authors concede that there were many studies that examined the long-term retention and performance of clinical procedures but which were excluded because they were not RCT. Educationalists would apply a more qualitative approach to learning that would include, for example, opinions and expectations of the undergraduates. Such use of qualitative research is highlighted by the authors in the discussion but they do not necessarily see this as good evidence. Other effects, such as students performing better when presented with a new learning environment, are also discussed. One area not considered was the quality of the CAL programmes themselves. The amount of interactivity can vary immensely between programmes and may be a factor in the subsequent success of the learning experience.

As a review of RCT of the effectiveness of CAL in the learning environment, this article produces an answer. Whether a RCT is the correct tool to evaluate the success of CAL is another question.

\section{Damien Walmsley}

School of Dentistry, University of Birmingham, Birmingham, UK

Evidence-Based Dentistry (2003) 4, 81.

doi:10.1038/sj.ebd.6400219 